\title{
Seismic damage simulation in urban areas based on a high-fidelity structural model and a physics engine
}

\author{
Zhen $\mathrm{Xu}^{\mathrm{a}}$, Xinzheng $\mathrm{Lu}^{\mathrm{a}}$, Hong Guan ${ }^{\mathrm{b}}, \mathrm{Bo} \mathrm{Han}^{\mathrm{a}}$ and Aizhu Ren ${ }^{\mathrm{a}}$ \\ ${ }^{a}$ Key Laboratory of Civil Engineering Safety and Durability of China Education Ministry, \\ Department of Civil Engineering, Tsinghua University, Beijing 100084, P.R. China. \\ ${ }^{\mathrm{b}}$ Griffith School of Engineering, Griffith University Gold Coast Campus, Queensland 4222, \\ Australia.
}

\begin{abstract}
Effective seismic damage simulation is an important task in improving earthquake resistance and safety of dense urban areas. There exist two significant technical challenges for realizing such a simulation: accurate prediction and realistic display. A high-fidelity structural model is proposed herein to accurately predict the seismic damage that was inflicted on a large number of buildings in an urban area via time-history analysis (THA), with which the local damage to different building stories is also explicitly obtained. The accuracy and efficiency of the proposed model are validated by a refined finite element (FE) analysis of a typical building. A physics engine-based algorithm is also proposed that realistically displays building collapse, thus overcoming the limitations of the high-fidelity structural model. Furthermore, a visualization system integrating the proposed model and collapse simulation is developed so as to completely display the seismic damage in detail. Finally, the simulated seismic damage of a real medium-sized Chinese city is evaluated to demonstrate the advantages of the proposed techniques, which can provide critically important reference information for urban disaster prevention and mitigation.
\end{abstract}

Keywords: Seismic damage; Urban areas; High-fidelity structural model; Collapse simulation; Physics engine; Realistic visualization.

\section{Introduction}

Urban cities, especially metropolitan areas, generally have a dense population 
and infrastructure. A strong earthquake may thus lead to a very large number of casualties and significant financial losses. Recently, powerful earthquakes have inflicted severe damage on several dense urban areas (Ye et al. 2010; Gonzalez-Huizar et al. 2012), e.g., the 2010 Chile Earthquake caused more than 500 deaths and a total damage thought to be in the range of 15 to 30 billion US dollars, which is approximately $9-18 \%$ of the country's total GDP (Lindell 2010; Lorito et al. 2011). Therefore, seismic damage simulation is an important task in improving earthquake resistance and safety of dense urban areas.

Seismic damage simulations of dense urban areas currently face two significant technical challenges: (1) how to accurately predict seismic damage to a large number of buildings and (2) how to realistically display the predicted earthquake disaster scenarios.

From the simplest single-degree-of-freedom (SDOF) model to the most complicated solid element model, various studies have been conducted with the objective of accurately predicting seismic damage to urban buildings (Tyagunov et al. 2006; Korkmaz 2009; Steelman and Hajjar 2009; Tang et al. 2011; Yamashita et al. 2011). Traditional prediction methods based on vulnerability matrices can no longer satisfy the accuracy requirements for the current seismic damage predictions. As such, the Federal Emergency Management Agency (FEMA) of the United States developed the HAZUS system and proposed Advanced Engineering Building Modules (AEBM), which are able to take the structural features of buildings and possible earthquake scenarios into account (FEMA-NIBS 1997, 1999; FEMA 2009, 2012a, b). Nevertheless, the highly local damage to different stories of a single building cannot be explicitly identified in HAZUS, because buildings are treated as SDOF systems. Wijerathne et al. (2013) proposed an "integrated earthquake simulation (IES)" to simulate the seismic damage of Tokyo city using nonlinear THA and refined structural models (e.g. a discrete element method (DEM) and fiber element model). However, the IES must be performed on a supercomputer due to the massive computational workload. Therefore, an accurate and efficient prediction method for seismic damage to a large number of buildings in urban areas still needs to be developed. 
It should be noted that when high-fidelity structural models are adopted, the task of simulating the collapse processes of a large number of buildings is extremely time-consuming. Building collapses have significant impact not only on attempts at realistic visualization, but also on rescue and transportation planning, because roads may very well be obstructed or obscured by the collapsed buildings. Therefore, a highly efficient collapse simulation methodology is needed for displaying building seismic damage.

Visualizations of seismic damage have been extensively studied by a large number of researchers (Duzgun and Yucemen 2011; Xu et al. 2008). For example, Abdalla and Tao (2005) created a 3D model of Vancouver City based on the distributed GIS and evaluated the extent of seismic damage to the entire city. However, the existing work mainly focuses on displaying the static earthquake disaster scenario instead of presenting the dynamic process of the collapse of buildings.

The "physics engine" is a recently introduced computer graphics technology that specializes in calculating the complex physical behaviors of objects, such as the spread of smoke, flow of water and rigid-body collisions (Millington 2007). Havok, Bullet and PhysX are the three most popular physics engines, and they are all widely used in visual simulations and the making of videos (Havok 2012; Game Physics Simulation 2012; NVIDIA 2012). This technology can be used to simulate the processes of the collapse of buildings or other structures (Boeing and Bräunl 2007; Joselli et al. 2008; Maciel et al. 2009). For instance, Xu et al. (2013) employed the physics engine to simulate the movement of debris in bridge collapses. Nevertheless, the physics engine has not yet been extensively applied to seismic damage simulations. Thus, utilization of a physics engine to simulate the seismic-induced collapse processes of buildings merits further investigation.

In this paper, solutions to the two aforementioned technical challenges in simulating seismic damage to a dense urban area are presented. A high-fidelity structural model based on the multi-story concentrated-mass shear (MCS) model is proposed for accurately predicting seismic damage to a large number of buildings in an urban area using THA. The accuracy and efficiency of the proposed model are 
validated through a refined $\mathrm{FE}$ analysis of a typical building. To realistically characterize earthquake disaster scenarios, an algorithm using the physics engine for visualizing earthquake-induced building collapses is also proposed, which overcomes the limitations of the high-fidelity structural model. Furthermore, a realistic visualization model of urban buildings is established and a complete visualization system integrating the high-fidelity structural model with collapse simulation is implemented to provide detailed seismic damage. Finally, the simulated seismic damage of a real medium-sized Chinese city is investigated to demonstrate the advantages of the proposed techniques, which can provide critically important reference information for urban disaster prevention and mitigation.

\section{High-fidelity structural model of seismic damage}

\subsection{The proposed MCS model}

The computational workload for the THA is massive when dealing with a large number of buildings in a dense urban area. To balance the computational workload and accuracy, the multi-story concentrated-mass shear (MCS) model is adopted for high-fidelity seismic damage analysis, as illustrated in Figure 1. The MCS model is a multi-degree-of-freedom model that is able to calculate the local damage to different stories with a high degree of efficiency (Hori and Ichimura 2008).

The accuracy of the MCS model is dominated by the inter-story hysteretic behavior model. The inter-story hysteretic model proposed by Han et al. (2012) (see Figure 2) is adopted in this study, as it is adaptive to most typical structural types and capable of simulating complicated inter-story behaviors (e.g. yielding, hardening, softening and pinching).

To determine the parameters in the inter-story hysteretic model, a method based on pushover analysis is proposed herein. All of the buildings within an urban area can be divided into different classes according to their different structural types (FEMA, 2012a) and typical buildings are selected from each class. Refined FE building models 
are then created for the selected typical buildings using the fiber beam element model and the multi-layer shell model (Lu et al. 2013; Guan and Loo 1997). The inter-story force-displacement relationships are obtained via a cyclic pushover analysis of the refined FE models. Simultaneously, a cyclic pushover analysis is also conducted on the corresponding MCS model created for the same building. The parameters of the hysteretic model in the MCS model are continuously modified until the two inter-story force-displacement relations agree with each other (Figure 3). In this study, a good agreement is considered to have been achieved if the errors of four individual key points (i.e. the peak, yielding, ultimate and pitching points) between the two inter-story relation curves are within $10 \%$.

Once the parameters of the inter-story hysteretic model of typical buildings have been determined, these parameters are mapped to the inter-story hysteretic model of all the remaining buildings in the same class as follows.

Step (1): According to the similar MCS modeling research conducted by Hori (2011), the initial inter-story stiffness and the mass of each story can be assumed to be uniformly distributed along the height of the building.

Step (2): The vibration periods and story mass of each building are calculated according to the empirical equations in the Load Code for the Design of Building Structure (GB 50009-2001) of China (MCPRC 2002), with which the initial inter-story stiffness are obtained.

Step (3): The lateral strength and deformation potential of the typical buildings are mapped to the remaining buildings in the same class in proportion to the inter-story stiffness, story mass and number of stories of the building (Hori 2011).

It should be noted that if the mass or stiffness distributions in a building vary significantly along the height, such a building is treated as a special case and its MCS model needs to be established directly based on the refined FE model for such a building, as shown in Figure 3. However, as pointed out by Hori (2011), most of the buildings in an urban area can be approximately modeled with the above parameter-mapping process.

Using the THA based on the aforementioned high-fidelity MCS model, the local 
seismic damage to different stories of each building in a dense urban area can be predicted accurately and the necessary data on seismic damage (e.g. the displacement, acceleration and inter-story drift ratios) can be subsequently obtained.

\subsection{Validation}

To validate the accuracy and efficiency of the MCS model, a 6-story reinforced concrete (RC) frame building is investigated herein, which is a very common type in most urban areas. Using the fiber beam elements and the multi-layer shell elements (Lu et al. 2013; Guan and Loo 1997), a refined FE model of the RC frame is established using the general purpose FE software MSC.Marc, as illustrated in Figure 4.

Through a cyclic pushover analysis, the lateral hysteretic curves of the refined FE model are obtained for each story. Based on these hysteretic curves, the inter-story hysteretic parameters of the MCS model are then determined. The lateral hysteretic behavior of the bottom story as a result of both the FE model and the MCS model are presented in Figure 5. It is evident that the two hysteretic curves agree very well with each other.

The nonlinear THA for the building is also performed for the refined FE model and the MCS model. The widely used El-Centro record is taken as the input ground motion (Chopra 1995) and a $600 \mathrm{~cm} / \mathrm{s}^{2}$ peak ground acceleration (PGA) is adopted. For the refined FE model, computation is terminated at $5.412 \mathrm{~s}$ due to the large nonlinear deformation which results in a non-convergent solution to the FE program. Before $5.412 \mathrm{~s}$, the top displacement values of the building versus the time histories predicted by the two models are presented in Figure 6. Again, an excellent agreement is achieved. Similar agreement is also found for the displacement of each story, as displayed in Figure 7. All of these comparisons confirm that the MCS model is able to reliably and accurately simulate the seismic behavior of a typical and representative frame building structure.

In terms of computational efficiency, the proposed techniques exhibit a 
predominant advantage. On a quad-core Xeon-based server (2.0 GHz per core), the above THA of the 6-story building structure takes more than 2 hours using the refined FE model, whereas less than 2 seconds using the MCS model.

\section{Realistic seismic damage visualization}

\subsection{Physics engine-based collapse simulation}

Using the high-fidelity MCS model, the computational workload required to implement the entire collapse simulation of each and every building in a dense urban area is impractically heavy. For each building, it is also extremely time-consuming to accurately compute large deformations and collisions between the different stories based on the MCS model. To solve this problem, a physics engine can be used as an ideal alternative means of simulating complex movement and interactive collisions in the process of building collapse, due to its substantial advantage in multi-body dynamics and collision detection. Furthermore, physics engines are highly efficient in large-scale simulation, e.g. the physics engine PhysX adopts a graphic process unit (GPU) to accelerate computation (NVIDIA 2012). As such, PhysX is used in this study for the simulation of the collapse of a large number of buildings.

To incorporate the results of the MCS model, this study considers a building story as a basic element in the collapse simulation, i.e. each story is modeled by a box-shaped rigid-body with the same area, height and mass. In addition, the ground is modeled as a planar rigid-body of infinite mass. In the proposed MCS model, building collapse is considered to have been initiated when its inter-story drift ratio reaches the collapse criterion specified in HAZUS (FEMA 2012a). This is presented in Figure 8a. To accurately simulate the building collapse, the displacement and velocity data for the different stories predicted by the MCS model when collapse occurs can be used as the initial state for the following collapse simulation by PhysX, as shown in Figure 8b. The subsequent movement of a rigid-body story under gravity is simulated by PhysX with an initial collapse displacement and velocity until it collides with other 
rigid-body stories or the ground. This is demonstrated in Figure 8c. Such a collapse simulation by PhysX provides a real-time visualization of the collapse process of a dense urban area and is much more efficient than any direct implementation with the MCS model.

To visualize the process of building collapse, PhysX must be integrated with a graphics engine. To fully control the rendering process, an open-source graphics engine OpenSceneGraph (OSG) is used as the visualization platform (OSG Community 2012). In OSG, all of the geometries are managed and stored by the Geode node (OSG Community 2012), whereas, in PhysX, all the objects in the physical calculations are represented by the Class Actor (NVIDIA 2012). To ensure an accurate correlation between the physical calculation and the graphical display, each building story is modeled as a Geode node in OSG, and is also modeled accordingly as an Actor in PhysX. The initial factors (e.g. the shape, initial position and velocity when collapse occurs) remain the same between the Actor and the Geode. In addition, a plain Static Actor, which is a special Actor with infinite mass in PhysX, is used to model the ground (NVIDIA 2012).

To dynamically display the calculation results of PhysX in OSG, the Callback mechanism, which implements the user-defined functions before rendering each visualization frame (OSG Community 2012) is adopted herein. In each frame, the movement data from Actors is transferred to the corresponding Geodes and the Geodes are updated by this data, in order to dynamically display the movement of each story (Xu et al. 2013). This continuous looping forms the visualization process of building collapse.

\subsection{Realistic visualization model of urban buildings}

A realistic visualization model of urban buildings is a critically important foundation for displaying seismic damage. The MCS model simplifies the building stories into concentrated-masses, resulting in a lack of full 3D façade information. Thus, the Geographic Information System (GIS) is introduced to provide the 
necessary geometric information (e.g. the site locations and the ground polygon) in order to create the 3D geometric models of the buildings (Xu et al. 2008). By extruding the ground polygons of buildings, the 3D geometric models of the buildings are created story-by-story based on the MCS model. Additionally, the multi-texture technology proposed by Tsai and Lin (2007) is adopted, which has the capacity of mapping different textures onto different faces of the building. The above procedure is illustrated in Figure 9.

\subsection{Integrated visualization system}

Based on the proposed techniques, the complete visualization process of seismic damage to buildings can be divided into two stages: the pre-collapse stage (i.e. the inter-story drift does not reach the collapse criterion) and the post-collapse stage.

In the pre-collapse stage, all the deformations of the building stories are determined by the nonlinear THA results of the MCS model, and each visualization frame is related to the corresponding time step in the THA. To display the dynamic deformations of the buildings, a new class called updater is created by inheriting the Class UpdateCallback that manages the geometry updating in the Callback mechanism (OSG Community 2012). During the Callback process for each frame, the updater uses the displacement data at the corresponding time step from the THA to update the vertices of the different stories. This continuous updating process enables a complete visualization of the deformation that occurs in the buildings. When the maximum time step is reached or collapse of the building occurs, the updater is destructed to release the memory.

In the post-collapse stage, the physical model of each building is created before rendering. If the collapse criterion is satisfied in the THA of the MCS model, the updater that links the graphic engine to the THA results is destructed. Simultaneously, the corresponding Actor is activated. The subsequent collapse movements of the building stories are calculated in real-time and presented according to the aforementioned technique of collapse simulation. The entire process covering the 
pre-collapse and post-collapse stages is illustrated in Figure 10.

\section{Application and discussion}

Shantou, a medium-sized city in China, is selected as the case study in the present work. The downtown region of Shantou has 7,449 buildings with a total of 43,016 building stories. The MCS models are created for all the buildings. The El Centro-1940 earthquake ground motion is chosen for the implementation of the nonlinear THA. Five damage states (i.e. none, slight, moderate, extensive and complete) are defined as indicated in HAZUS (FEMA 2012b), and the inter-story drift ratio predicted by the MCS model is used to evaluate these damage states. The seismic damage to the buildings across the entire region is illustrated in Figure 11 and a selected local area is enlarged to show the detailed seismic damage inflicted on the different stories (Figure 12).

Compared to the existing models (such as HAZUS), the MCS model that was adopted in this study can explicitly provide local damage inflicted on different stories, as evident in Figure 12. It not only provides a more accurate seismic damage result, but also detailed data that enables substantial improvement on the visualization of earthquake scenarios. Furthermore, although the high-fidelity MCS model is used, the required computation time was less than 10 minutes on a desktop computer (i5 processor, $2.8 \mathrm{GHz}$; $4 \mathrm{~GB}$ memory); therefore, the proposed model is notably efficient.

Based on the results of the MCS models and the GIS data, realistic building visualization models are established (Figure 13a), and amplified building deformation is provided in Figure 13b. Due to the massive number of buildings in this urban area, a simulated collapse of all the buildings using the high-fidelity structural model cannot be accomplished using conventional desktop computers. However, by introducing PhysX, the collapse process can be presented in real-time on the same desktop computer with a speed of 30 FPS (frame per second), as illustrated in Figures $13 \mathrm{c}$ and $13 \mathrm{~d}$. Compared to the existing methods of visualizing seismic damage, the 
physics engine-based technique is able to present a more realistic process of building collapse that overcomes the existing shortcomings of the high-fidelity structural model.

The resulting visualization using the physics engine can be used for emergency decision makings on issues of transportation and rescue. This is because the detailed visualization results (e.g. the collapse direction and the location of falling materials) provide important information as whether the collapsed buildings obstruct the surrounding road networks. Further, the outcome of this study can also be used in virtual drills on escape and rescue in earthquakes. By using the two digital light processing (DLP) projectors and double monitors, a stereo visualization of an earthquake disaster is performed for Shantou City, and the terrain and sky are added to the scenario of the earthquake disaster simulation (Figure 14). The stereo visualization of the seismic damage creates a highly immersed and realistic earthquake scenario for emergency drilling.

\section{Conclusions}

A high-fidelity MCS structural model is proposed to be able to simulate seismic damage to a large number of buildings in a dense urban area via THA. Compared to the existing methods, the proposed MCS model can explicitly obtain the local damage values in different stories, which provides a more accurate prediction of seismic damage and also more detailed data for the purpose of a visualization of an earthquake scenario. Through a refined FE analysis of a typical RC frame building, the proposed model is validated to be both accurate and efficient in the simulation of seismic damage.

Compared to the existing methods, the physics engine-based technique enables both a more realistic and efficient simulation of building collapses and overcomes the shortcomings of the high-fidelity structural model. The dynamic visualization system integrating the high-fidelity structural model and collapse simulation is able to completely display seismic damage in detail and in real time. 
The investigation of a real Chinese medium-sized city demonstrates that the proposed techniques can be used for emergency decision makings on issues such as transportation and rescue and also in carrying out emergency drilling, which provides critically important reference information and technical support for urban disaster prevention and mitigation.

\section{Acknowledgements}

The authors are grateful for the financial support received from the National Key Technology R\&D Program (No. 2013BAJ08B02), the National Nature Science Foundation of China (Nos. 51308321, 51178249, 51222804), the Tsinghua University Initiative Scientific Research Program (No. 2012THZ02-2) and China Postdoctoral Science Foundation (2013M530632).

\section{Reference}

Abdalla R, Tao V (2005) Integrated distributed GIS approach for earthquake disaster modelling and visualization. In: Geo-Information for Disaster Management, Springer, Berlin, pp $1183-1192$

Boeing A, Bräunl T (2007) Evaluation of real-time physics simulation systems. In: Proceedings of 5th International Conference on Computer Graphics and Interactive Techniques in Australia and Southeast Asia, ACM, New York, pp 281-288

Chopra AK (1995) Dynamics of structures. Prentice Hall, New Jersey

Duzgun HSB, Yucemen MS, Kalaycioglu HS (2011) An integrated earthquake vulnerability assessment framework for an urban areas. Nat Hazards 59: 917-947

Federal Emergency Management Agency-National Institute of Building Sciences (FEMA-NIBS) (1997) Earthquake loss estimation methodology-HAZUS97.Washington, D.C.

Federal Emergency Management Agency-National Institute of Building Sciences (FEMA-NIBS) (1999) Earthquake loss estimation methodology-HAZUS99. Washington, D.C.

Federal Emergency Management Agency (FEMA) (2009) FEMA P695-Quantification of building seismic performance factors, Washington, D.C.

Federal Emergency Management Agency (FEMA) (2012a) Multi-hazard loss estimation methodology, earthquake model, HAZUS-MH 2.1 technical manual, Washington, D.C.

Federal Emergency Management Agency (FEMA) (2012b) Multi-hazard loss estimation 
methodology HAZUS-MH 2.1 advanced engineering building module (AEBM) technical and user's manual, Washington, D.C.

Game Physics Simulation (2012) Bullet physics library. http://www.bulletphysics.com

Gonzalez-Huizar H, Velasco AA, Peng ZG, Castro RR (2012) Remote triggered seismicity caused by the 2011, M9.0 Tohoku-Oki, Japan earthquake. Geophys Res Lett 39: L10302

Guan H, Loo YC (1997) Layered finite element method in cracking and failure analysis of beams and beam-column-slab connections. Struct Eng and Mech - An Int J 5:645-662

Han B, Lu XZ, Xu Z (2012) Urban regional seismic damage prediction based on GPU-CPU hybrid computing. In: Proceedings of 15th World Conference in Earthquake Engineering, Lisbon, Portugal

Havok (2012) Havok physics. http://www.havok.com/products/physics

Hori M, Ichimura T (2008) Current state of integrated earthquake simulation for earthquake hazard and disaster. J Seismol 12:307-321

Hori M (2011) Introduction to computational earthquake engineering (2nd ed.). Imperial College Press, London

Joselli M, Clua E, Montenegro A, Conci A, Pagliosa P (2008) A new physics engine with automatic process distribution between CPU-GPU. In: Proceedings of 2008 ACM SIGGRAPH symposium on Video games, ACM, New York, pp 149-156

Korkmaz KA (2009) Seismic safety assessment of unreinforced masonry low-rise buildings in Pakistan and its neighborhood. Nat Hazards Earth Syst Sci 9:1021-1031

Lindell MK (2010) Built-in resilience. Nat Geosci 3:739-740

Lorito S, Romano F, Atzori S et al (2011) Limited overlap between the seismic gap and coseismic slip of the great 2010 Chile earthquake. Nat Geosci 4: 173-177

Lu X, Lu XZ, Guan H, Ye LP (2013) Collapse simulation of reinforced concrete high-rise building induced by extreme earthquakes. Earthq Eng Struct D 42:705-723

Maciel A, Halic T, Lu Z, Nedel LP, De S (2009) Using the PhysX engine for physics-based virtual surgery with force feedback. Int J Med Robot Comp 5:341-353

Millington I (2007) Game Physics Engine Development (The Morgan Kaufmann Series in Interactive 3D Technology). Morgan Kaufmann Publishers Inc., San Francisco

Ministry of Construction of the People's Republic of China (MCPRC) (2002) Load code for the design of building structure (GB 50009-2001). China Architecture \& Building Press, Beijing, China

NVIDIA Corporation (2012) PhysX. http://www.nvidia.cn/object/physx_new_cn.html.

OSG Community (2012) OpenSceneGraph. http://www.openscenegraph.org/projects/osg/ 
Steelman J, Hajjar J (2009) Influence of inelastic seismic response modeling on regional loss estimation. Eng Struct 31: 2976-2987

Tang BX, Lu XZ, Ye LP, Shi W (2011) Evaluation of collapse resistance of RC frame structures for Chinese schools in seismic design categories B and C. Earthq Eng Eng Vib 10: 369-377

Tsai F, Lin HC (2007) Polygon-based texture mapping for cyber city 3D building models. Int J Geogr Inf Sci 21: 965-981

Tyagunov S, Grunthal G, Wahlstrom R, Stempniewski L, Zschau J (2006) Seismic risk mapping for Germany. Nat Hazards Earth Syst Sci 6: 573-586

Wijerathne M, Hori M, Kabeyazawa T, Ichimura T (2013) Strengthening of parallel computation performance of integrated earthquake simulation. J Comput Civil Eng 27: 570-573

Xu F, Chen XP, Ren AZ, Lu XZ (2008) Earthquake disaster simulation for an urban area with GIS, CAD, FEA, and VR integration. Tsinghua Science and Technology 13:311-316

Xu Z, Lu XZ, Guan H, Ren AZ (2013) Physics engine-driven visualization of deactivated elements and its application in bridge collapse simulation. Automat Constr 35: 471-481

Yamashita T, Kajiwara K, Hori M (2011) Petascale computation for earthquake engineering. Comput Sci Eng 13:44-49

Ye LP, Lu XZ, Li Y (2010) Design objectives and collapse prevention for building structures in mega-earthquake. Earthq Eng Eng Vib 9:189-200 


\section{Figure captions:}

Fig. 1. The multi-story concentrated-mass shear model for a building

Fig. 2. The inter-story hysteretic model (Han et al. 2012)

Fig. 3. Method for determining the parameters of hysteretic model in selected typical buildings

Fig. 4. The refined FE model of the 6-story RC frame building

Fig. 5. Lateral hysteretic behavior of the FE model and the MCS model in the bottom story

Fig. 6. Top displacement versus time histories predicted by the FE model and the MCS model

Fig. 7. Displacements of each story predicted by the FE model and the MCS model at $5.412 \mathrm{~s}$

Fig. 8. The process of collapse simulation in physics engine

Fig. 9 Procedure for creating a realistic visualization model of a building

Fig. 10. Complete process of visualization of seismic damage to a building

Fig. 11. Seismic damage to buildings of the Shantou city

Fig. 12. Seismic damage to buildings of a selected local area

Fig. 13. Visualization of seismic damage to buildings in a local area

Fig. 14. Stereo visualization of seismic damage for the Shantou city 

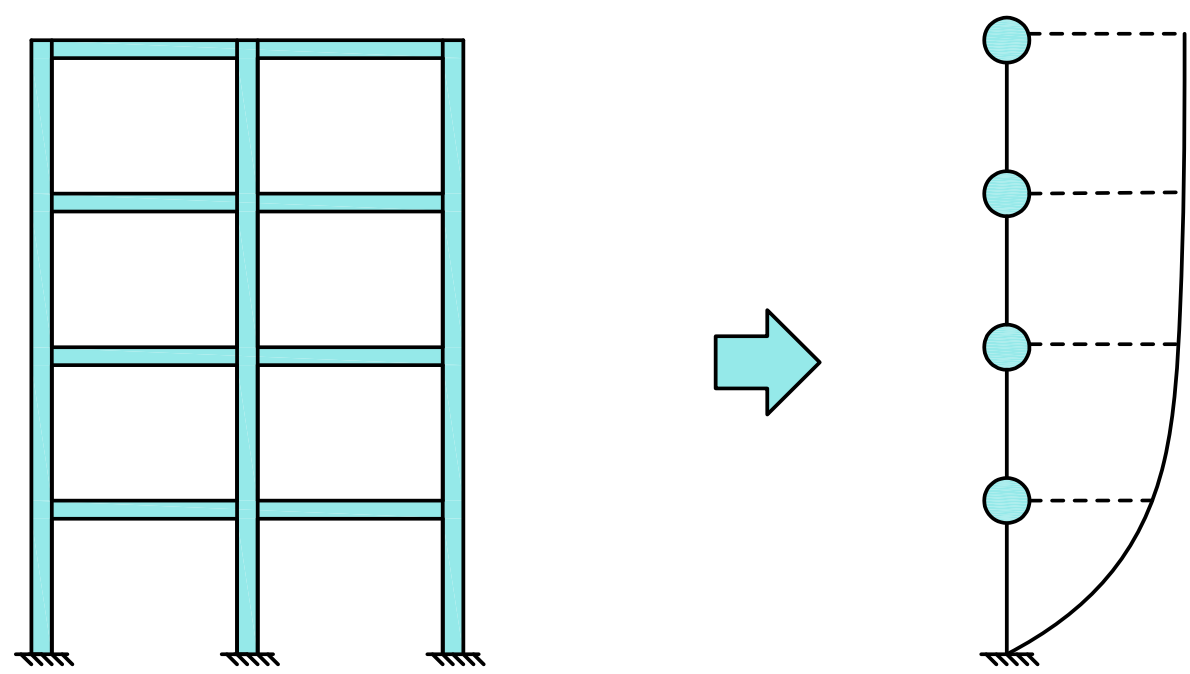

Fig. 1. The multi-story concentrated-mass shear model for a building 


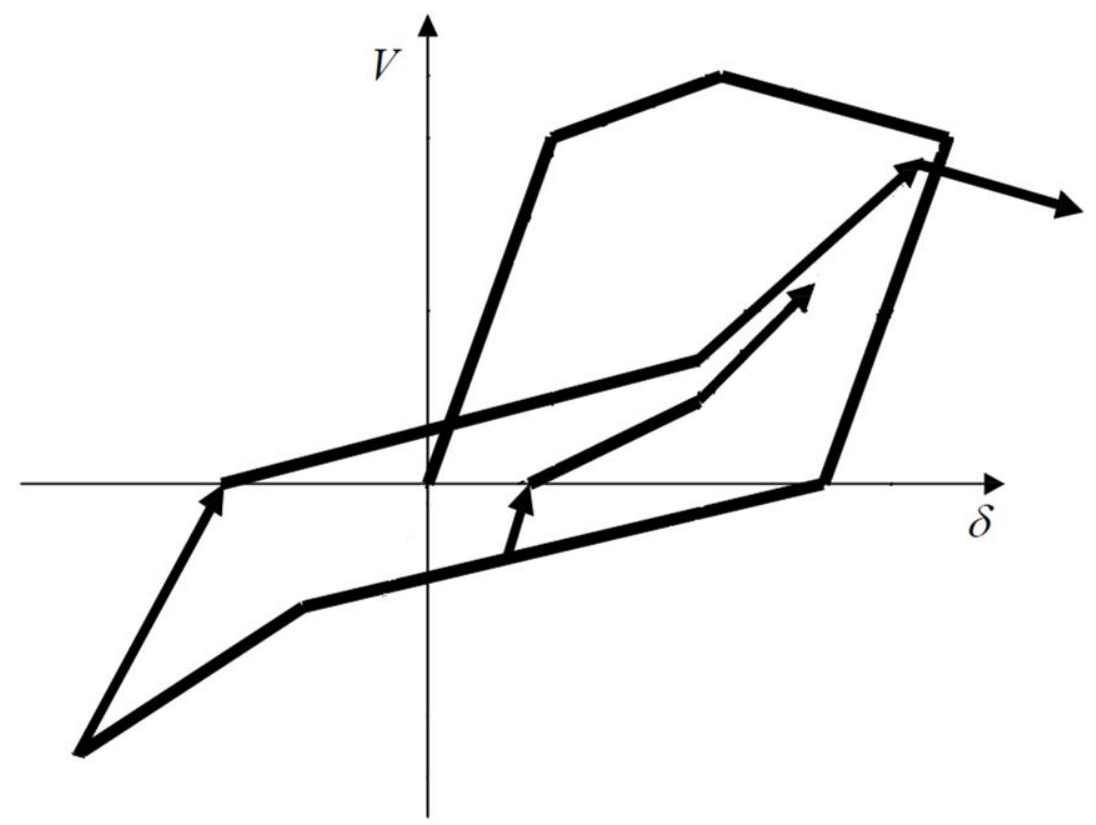

Fig. 2. The inter-story hysteretic model (Han et al. 2012) 


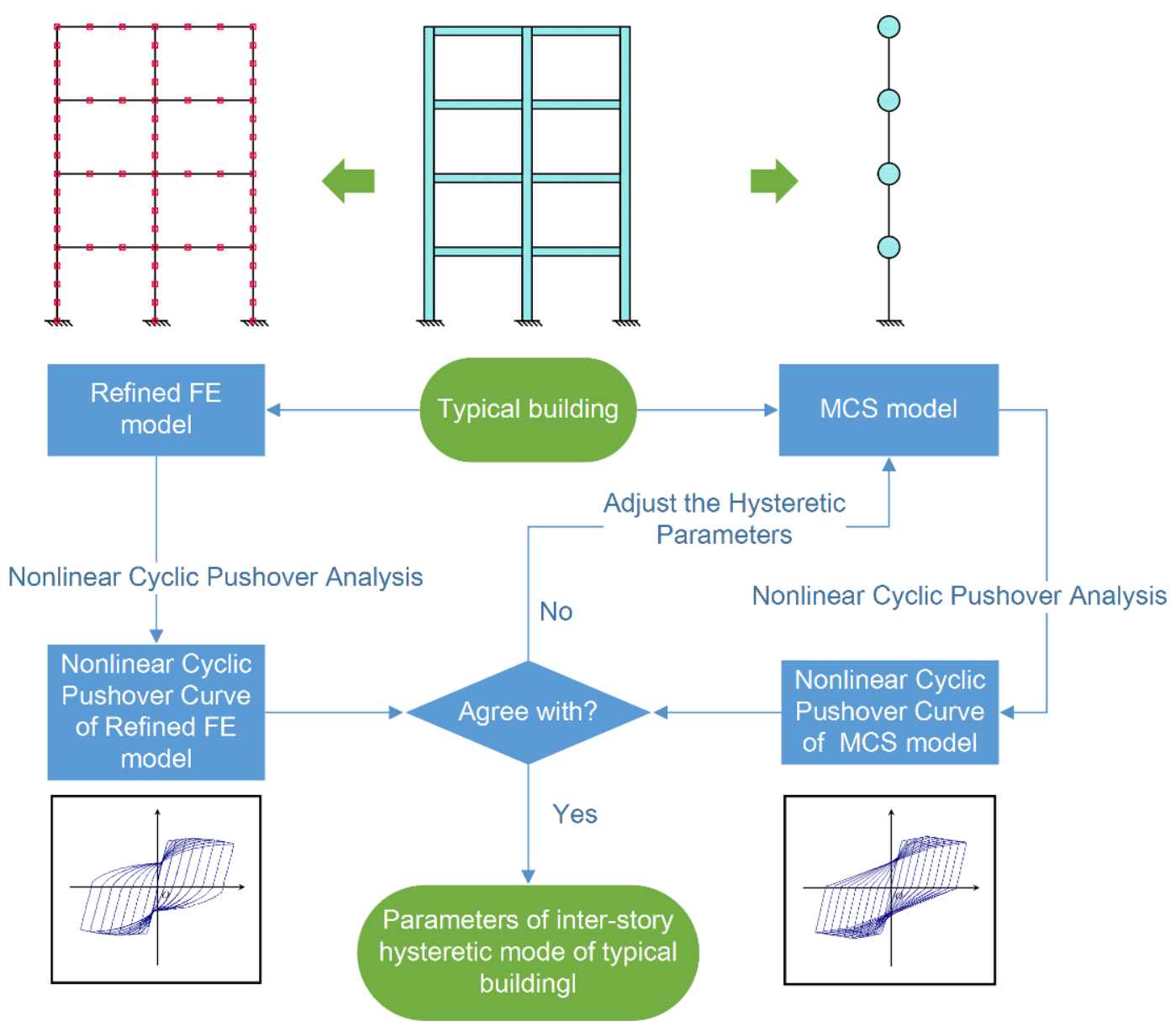

Fig. 3. Method for determining the parameters of hysteretic model in selected typical buildings 


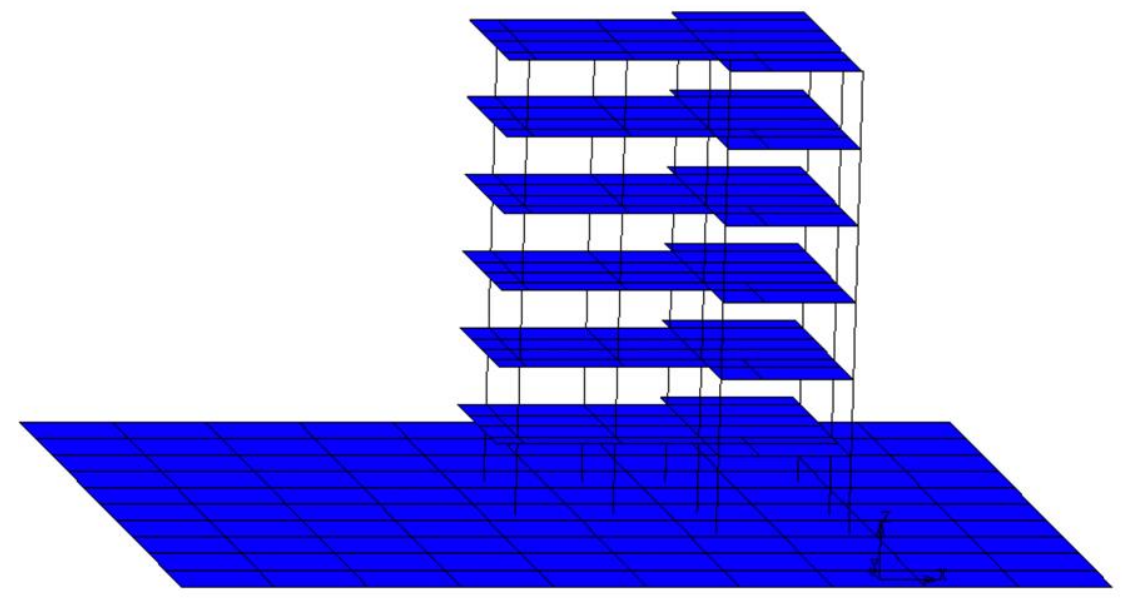

Fig. 4. The refined FE model of the 6-story RC frame building 


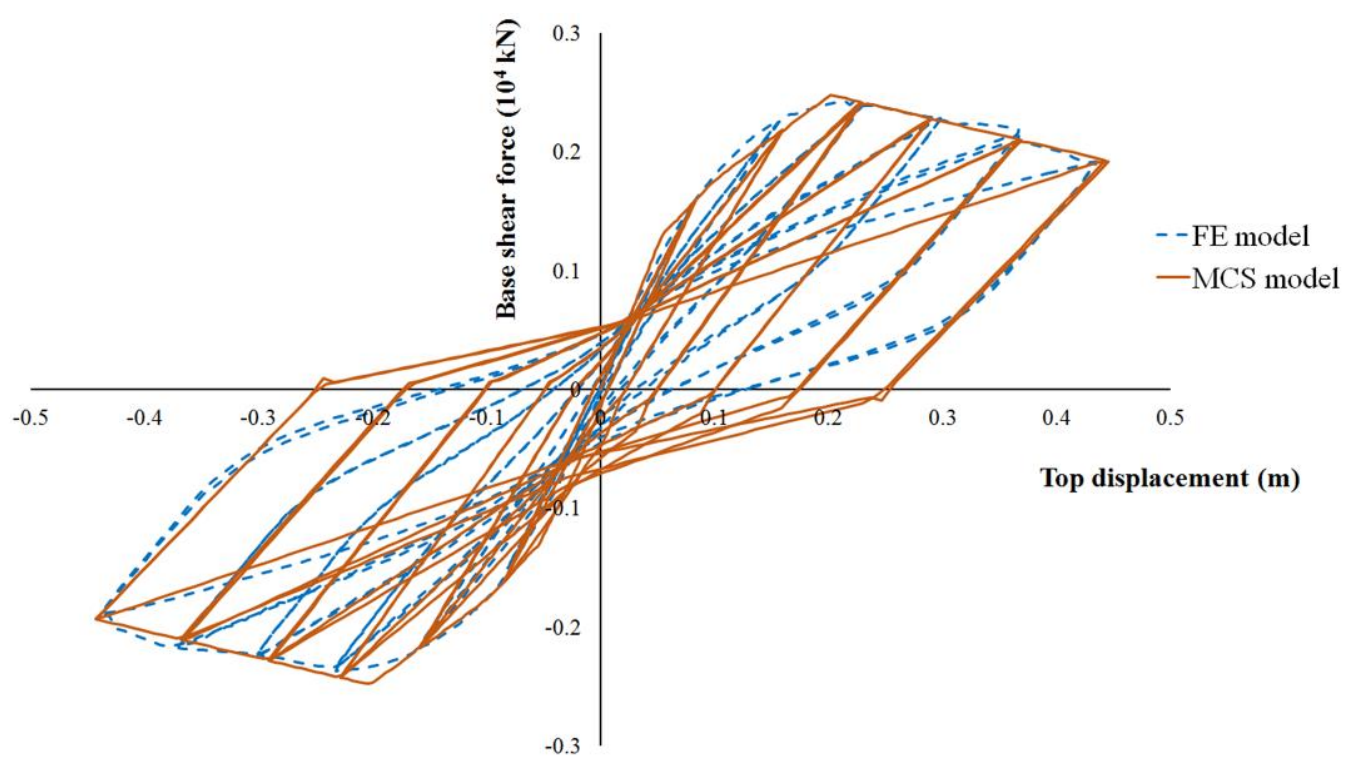

Fig. 5. Lateral hysteretic behavior of the FE model and the MCS model in the bottom story 


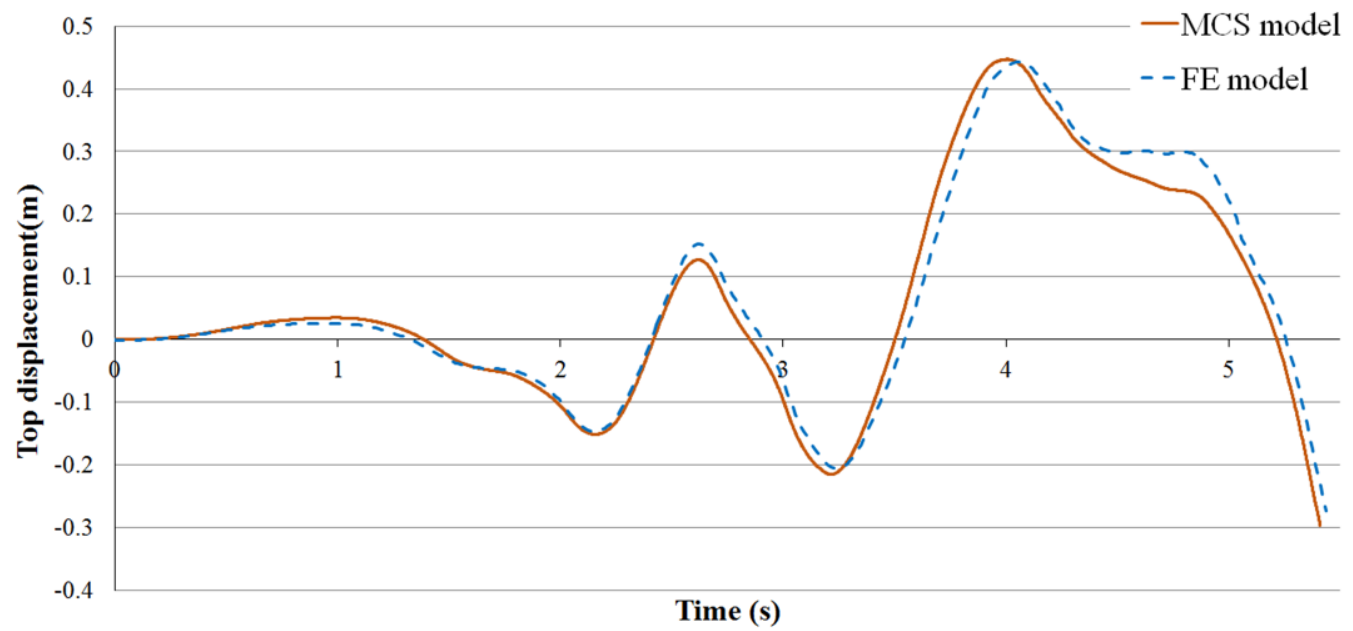

Fig. 6. Top displacement versus time histories predicted by the FE model and the MCS model 


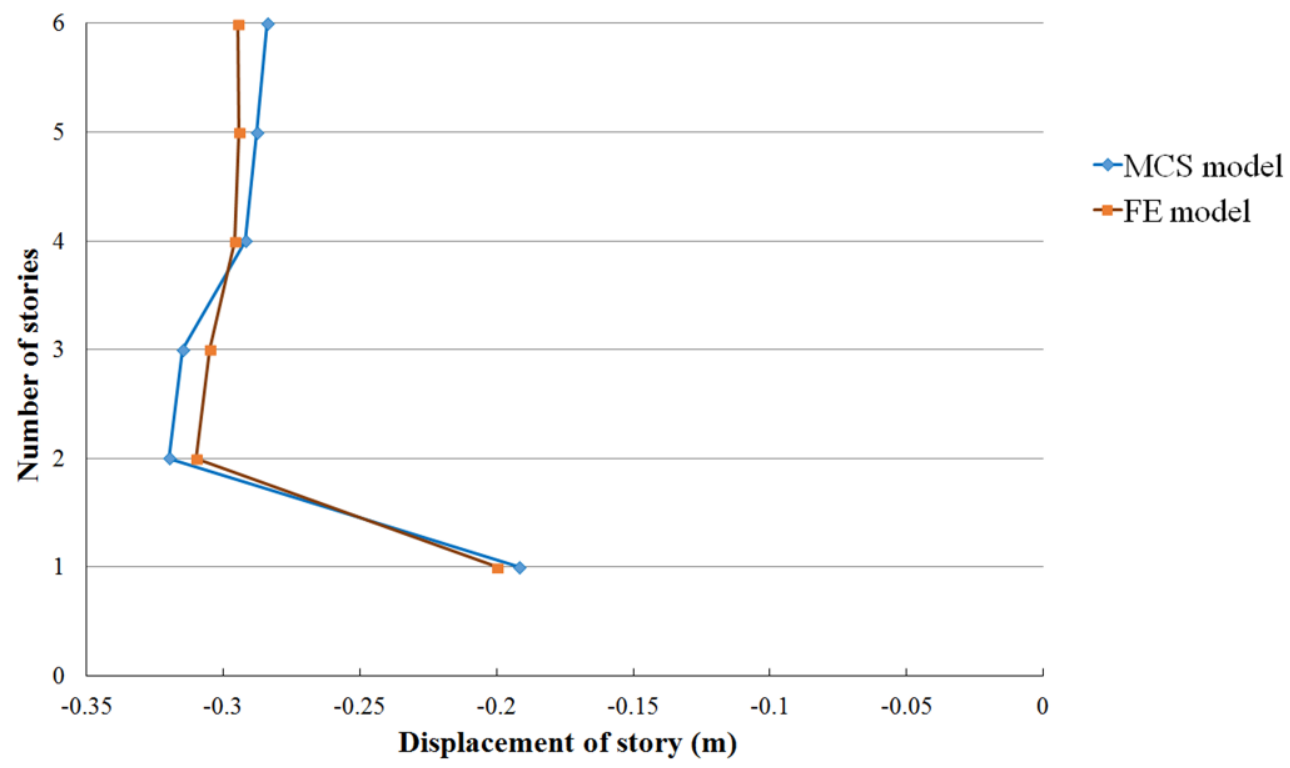

Fig. 7. Displacements of each story predicted by the FE model and the MCS model at $5.412 \mathrm{~s}$ 


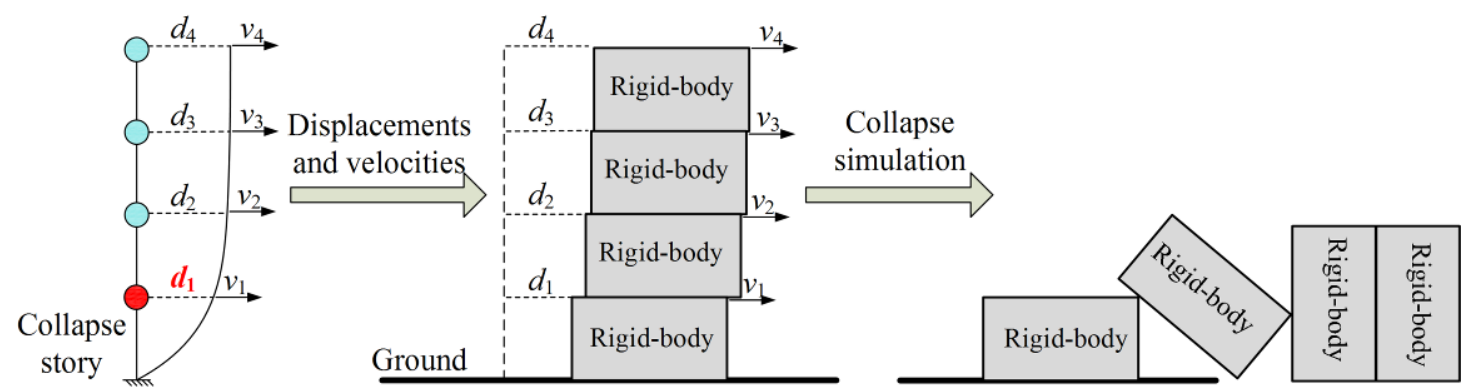

(a) Collapse state in MCS model

(b) Initial state in PhysX

(c) End state in PhysX

Fig. 8. The process of collapse simulation in physics engine 

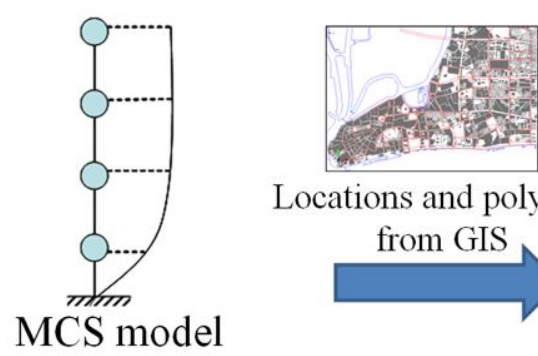

Locations and polygons from GIS
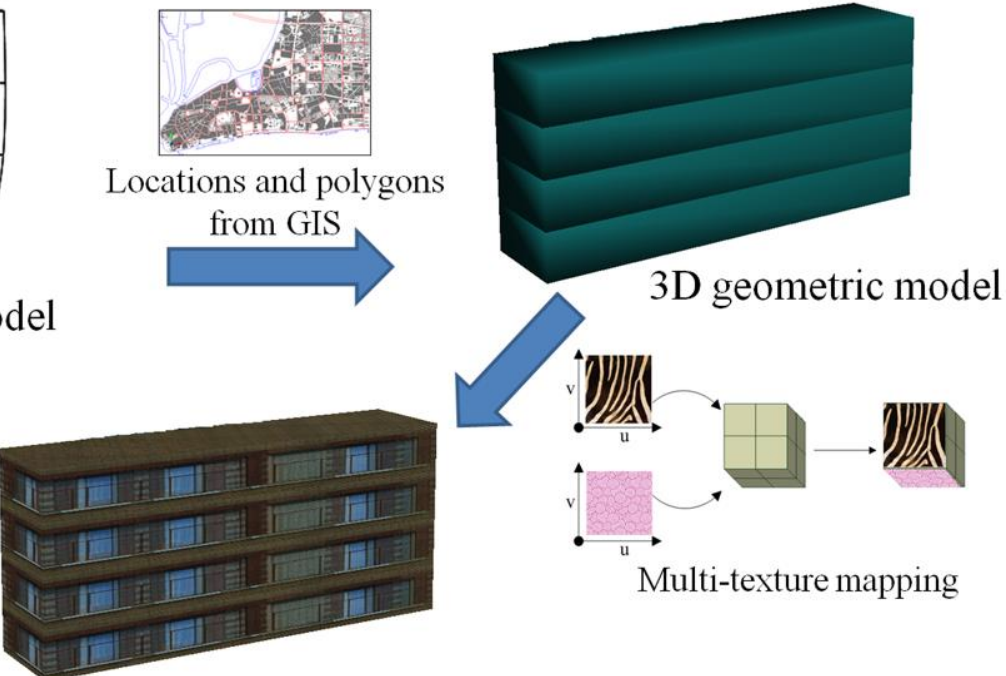

Realistic visualization model

Fig. 9 Procedure for creating a realistic visualization model of a building 


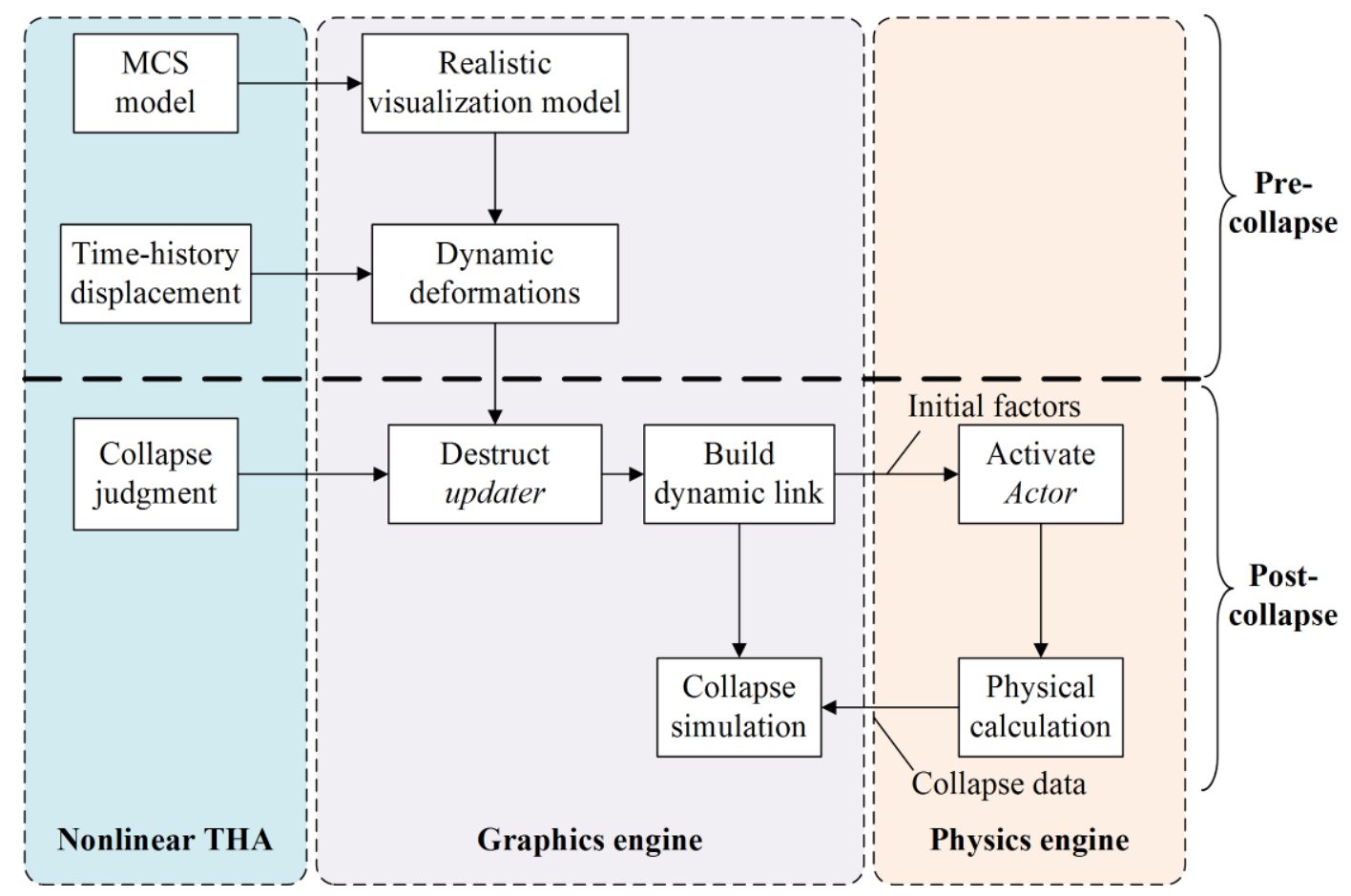

Fig. 10. Complete process of visualization of seismic damage to a building 


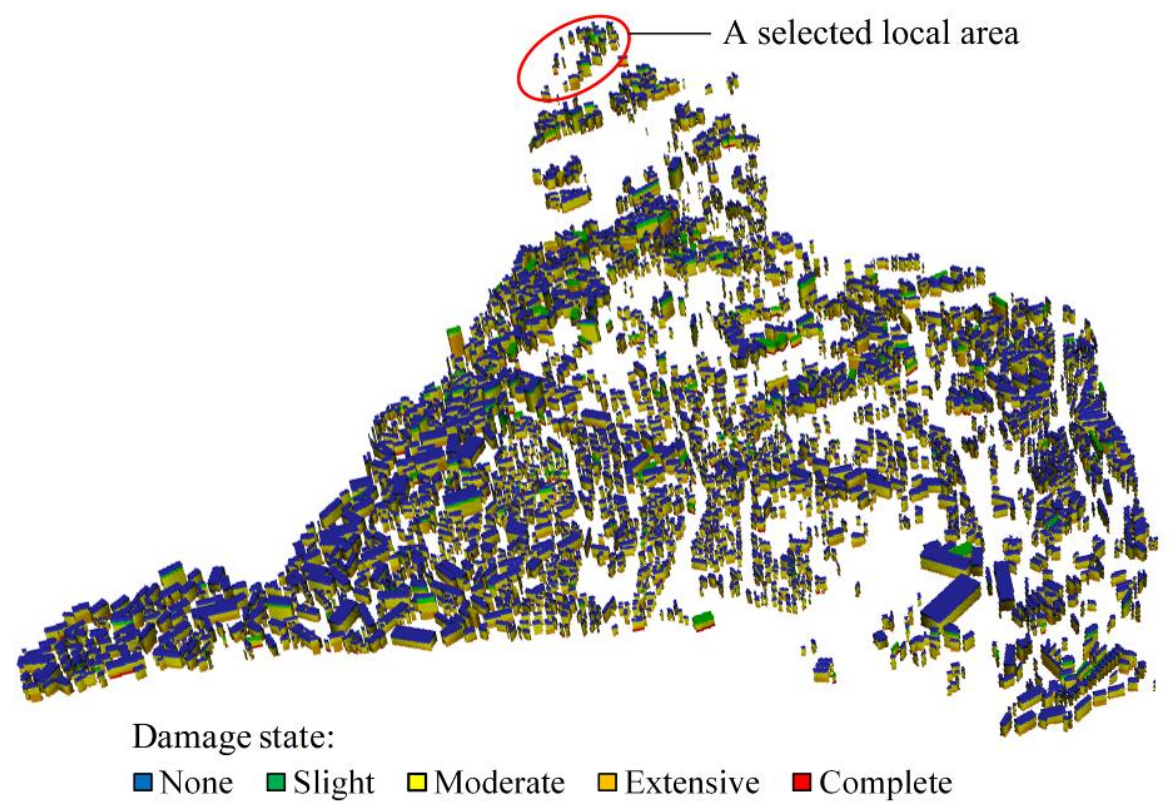

Fig. 11. Seismic damage to buildings of the Shantou city 


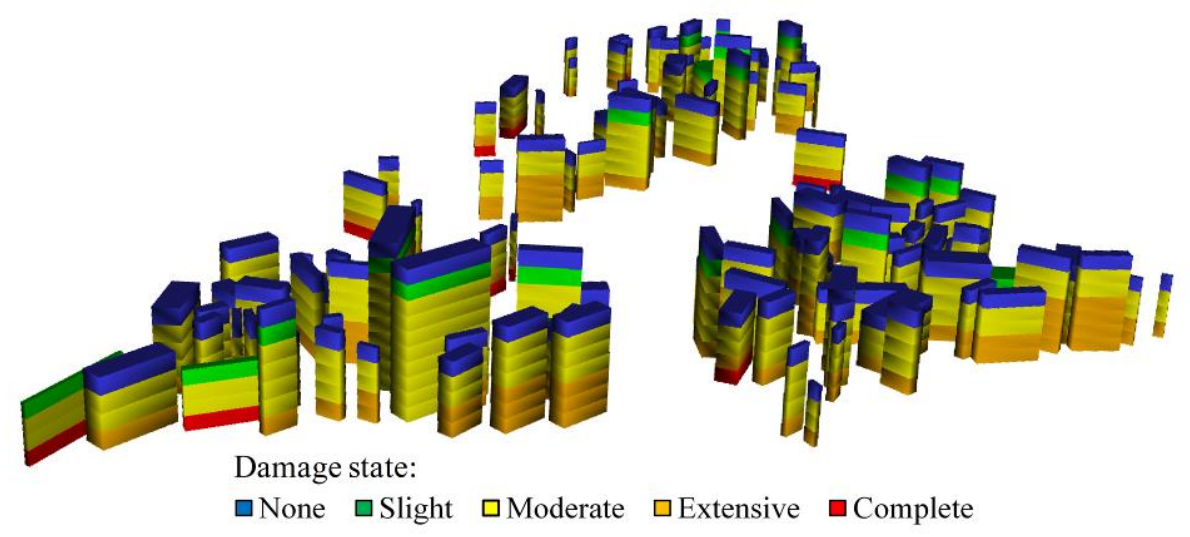

Fig. 12. Seismic damage to buildings of a selected local area 


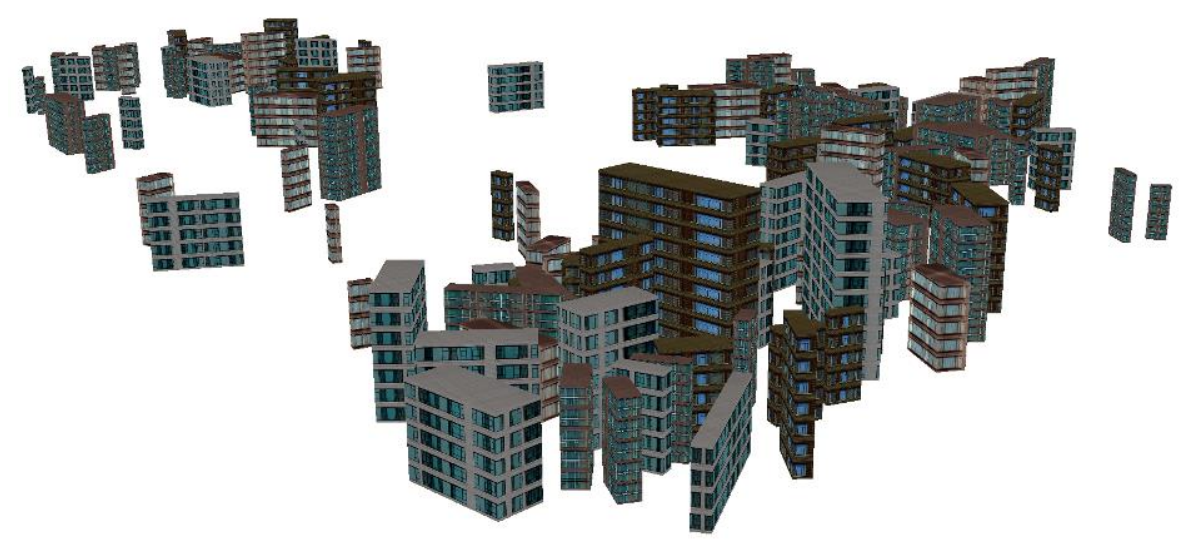

(a) Realistic building visualization model

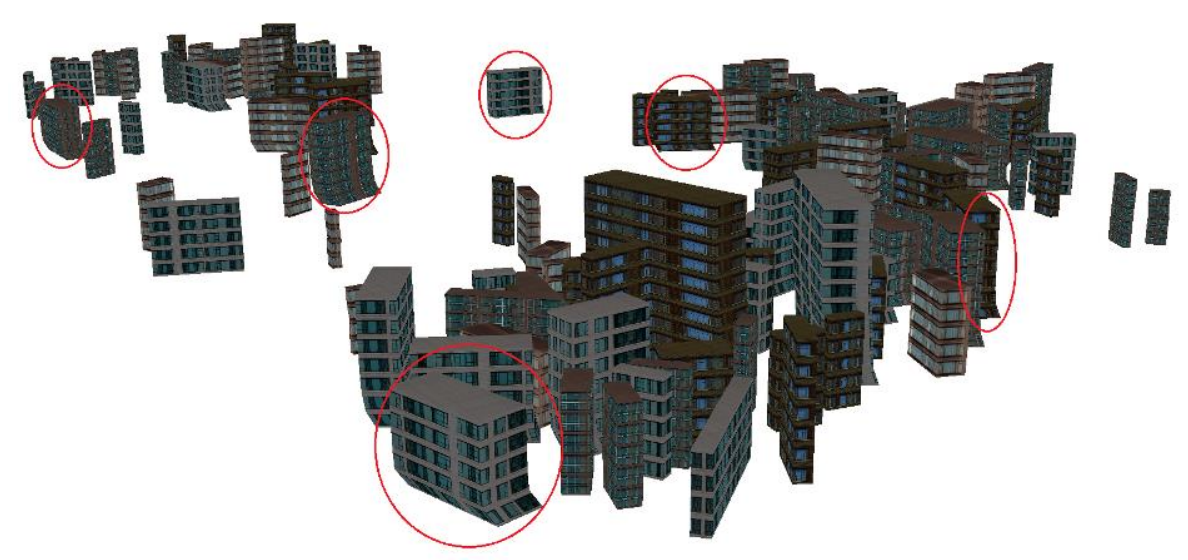

(b) Amplified deformation of the buildings (marked with circles) 


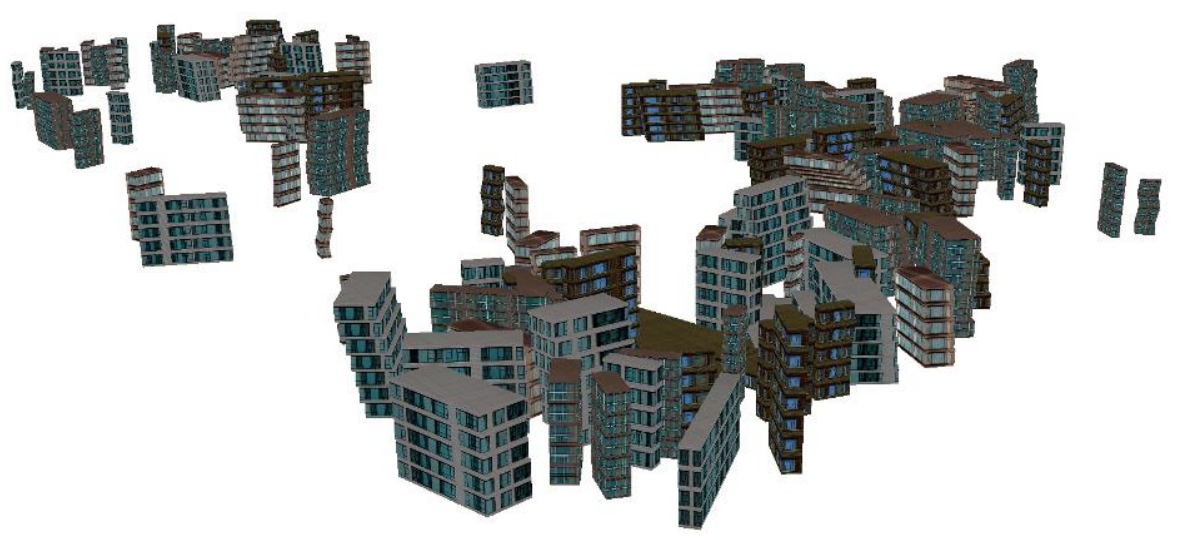

(c) Process of collapse

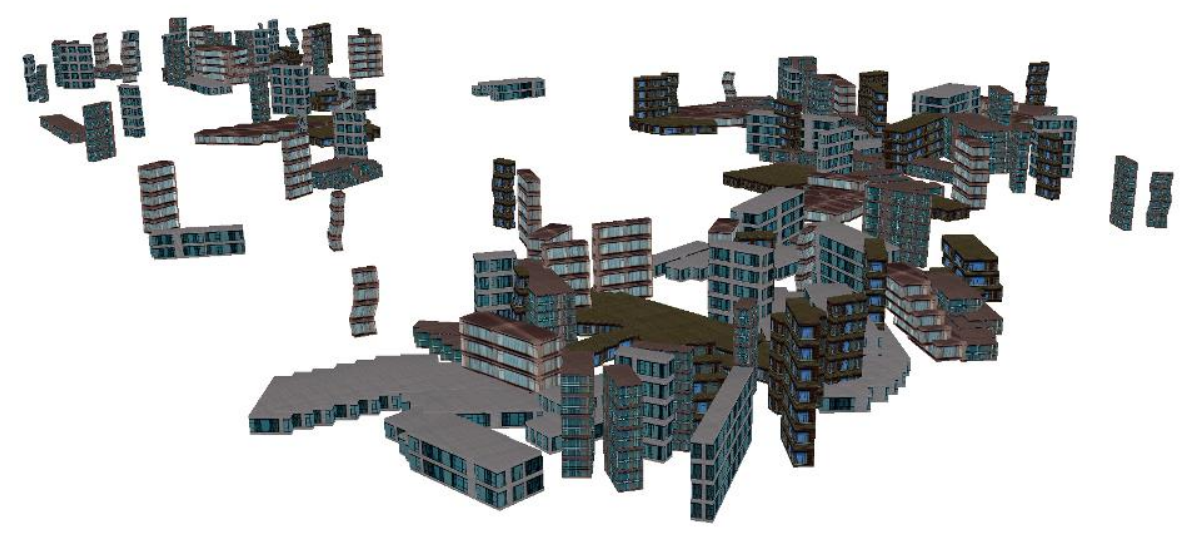

(d) End of collapse

Fig. 13. Visualization of seismic damage to buildings in a local area 

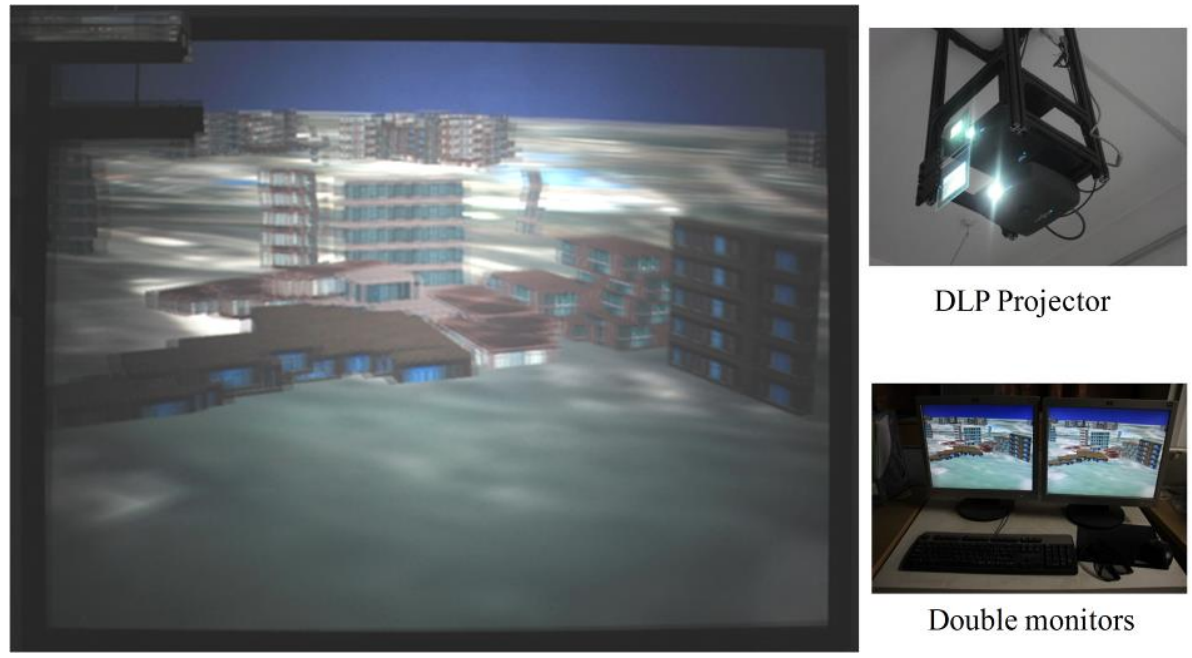

DLP Projector

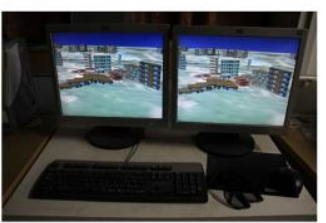

Double monitors

Fig. 14. Stereo visualization of seismic damage for the Shantou city 